\title{
NULL EXACT CONTROLLABILITY OF THE PARABOLIC EQUATIONS WITH EQUIVALUED SURFACE BOUNDARY CONDITION
}

ZHONGQI YIN

Received 25 January 2005; Revised 21 April 2005; Accepted 26 April 2005

This paper is devoted to showing the null exact controllability for a class of parabolic equations with equivalued surface boundary condition. Our method is based on the duality argument and global Carleman-type estimate for a parabolic operator.

Copyright (c) 2006 Zhongqi Yin. This is an open access article distributed under the Creative Commons Attribution License, which permits unrestricted use, distribution, and reproduction in any medium, provided the original work is properly cited.

\section{Introduction}

Let $T>0, \Omega \subset \mathbb{R}^{n}(n \in \mathbb{N})$ be a given bounded domain, $\partial \Omega=\Gamma_{0} \cup \Gamma_{1}\left(\Gamma_{1} \neq \varnothing\right)$ (where $\Gamma_{0}$ is the interior boundary and $\Gamma_{1}$ the outer boundary), $\Gamma_{0} \cap \Gamma_{1}=\varnothing$. For simplicity, we assume that $\Gamma_{0}, \Gamma_{1} \in C^{\infty}$ and $\omega \neq \varnothing$ is a given subdomain of $\Omega$. Denote the characteristic function of $\omega$ by $\chi_{\omega}$, and the unit outward normal vector of $\Omega$ by $\left(n_{1}, \ldots, n_{n}\right)$. Put $Q=$ $\Omega \times(0, T), Q^{\omega}=\omega \times(0, T)$, and $\Sigma=\partial \Omega \times(0, T)$. Let $a_{i j}(x) \in C^{2}(\bar{\Omega})$ satisfy $a_{i j}=a_{j i}$, and for some $\Lambda>0$, it holds that

$$
\sum_{i, j} a_{i j} \xi_{i} \xi_{j} \geq \Lambda|\xi|^{2}, \quad \forall(x, \xi) \in \Omega \times \mathbb{R}^{n}
$$

Here and henceforth, we denote $\sum_{i, j=1}^{n}$ simply by $\sum_{i, j}$.

We consider the following controlled parabolic equation with equivalued surface boundary condition:

$$
\begin{gathered}
\frac{\partial y}{\partial t}-\sum_{i, j} \frac{\partial}{\partial x_{i}}\left(a_{i j}(x) \frac{\partial y}{\partial x_{j}}\right)=\chi_{\omega}(x) b \quad \text { in } Q, \\
\left.y\right|_{\Gamma_{1}}=0,\left.\quad y\right|_{\Gamma_{0}}=m(t), \\
\int_{\Gamma_{0}} \frac{\partial y}{\partial n_{A}} d s=0, \quad y(x, 0)=y_{0}(x) \quad \text { in } \Omega,
\end{gathered}
$$

Hindawi Publishing Corporation

Journal of Applied Mathematics and Stochastic Analysis

Volume 2006, Article ID 62694, Pages 1-10

DOI 10.1155/JAMSA/2006/62694 
where $y=y(x, t)$ is the state, $b=b(x, t)$ is the control, $m(t) \in L^{2}(0, T)$ is an unknown function which depends only on the time variable $t, y_{0}$ is the initial state, and

$$
\frac{\partial y}{\partial n_{A}}=\sum_{i, j} a_{i j}(x) \frac{\partial y}{\partial x_{j}} n_{i} .
$$

In system (1.2), the state space is chosen as $L^{2}(\Omega)$, and the control space is $L^{2}(\omega)$. Let

$$
Y \triangleq\left\{y \in C\left([0, T] ; L^{2}(\Omega)\right) \bigcap L^{2}\left(0, T ; H^{1}(\Omega)\right)|y|_{\Gamma_{1}}=0,\left.y\right|_{\Gamma_{0}}=m(\cdot) \in L^{2}(0, T)\right\} .
$$

It can be shown that for any $y_{0} \in L^{2}(\Omega)$ and $b \in L^{2}(\omega \times(0, T))$, system (1.2) admits one and only one weak solution $y \in Y$ (cf. $[5,6,8])$.

The null exact controllability problem of (1.2) is formulated as follows: for any given $y_{0} \in L^{2}(\Omega)$, find a control $b(x, t) \in L^{2}(\omega \times(0, T))$ (if possible) such that the weak solution $y(\cdot) \in Y$ satisfies $y(T)=0$.

There are many concrete physical backgrounds for problem (1.2), for example, the problem of resistivity well logging, the unstable temperature field around an underground electric cable, and so on (cf. $[5,6])$.

In recent years, great progress has been made in the exact controllability problem of the linear and semilinear partial differential equations with Dirichlet or Neumann boundary condition, or other sorts of pointwise boundary value conditions $([1-4,7,9]$, and the references cited therein). However, to the author's best knowledge, there is no reference devoted to the same problem for the parabolic equations but with a spatial nonlocal boundary condition. In this paper, we will show the null exact controllability for system (1.2). By duality, the problem is reduced to the obtention of an observability inequality for the corresponding adjoint equation, which in turn is derived by means of a global Carleman-type estimate. Our method is stimulated by that in [4].

The rest of this paper is organized as follows. In Section 2, we state some preliminary results and our main results. The final section, Section 3 , is devoted to the proof of our main theorem.

\section{Main result}

Throughout this paper, $C$ denotes a positive constant depending only on $\lambda, \mu, \Omega, T$, and $\omega$, which may change from line to line.

To begin with, we fix $\omega_{0}$ to be a nonempty open subset of $\Omega$ such that $\bar{\omega}_{0} \subset \omega$. Let $\psi \in C^{\infty}(\bar{\Omega})$ satisfy $\psi>0$ in $\Omega, \psi=0$ on $\partial \Omega$, and $|\nabla \psi(x)|>0$ for all $x \in \Omega_{0}=\Omega \backslash \omega_{0}$. The existence of function $\psi$ was proved in [7]. In this paper, we further assume that the following technical condition holds:

$$
\left.\left(\frac{\partial \psi}{\partial n}\right)^{2} \sum_{i, j} a_{i j} n_{i} n_{j}\right|_{\Gamma_{0}}=\text { Const. }
$$

This technical condition admits several interesting cases such as $\Omega=\left\{x \in \mathbb{R}^{n}|r<| x \mid<R\right\}$ 
for some $0<r<R<\infty, a_{i j}(x)=\delta_{i}^{j}$, and $\partial \psi / \partial n=$ Const on $\Gamma_{0}$. In this case, one may choose $\psi(x)=\left(|x|^{2}-r^{2}\right)\left(R^{2}-|x|^{2}\right)$.

The main result in this paper is stated as follows.

Theorem 2.1. Under the assumption (2.1), system (1.2) is null exact controllable.

By means of the usual duality argument (see, e.g., $[3,9]$ ), the proof of Theorem 2.1 is easily reduced to the obtention of an observability inequality for the following adjoint system of system (1.2):

$$
\begin{gathered}
-L u \equiv-\frac{\partial u}{\partial t}-\sum_{i, j} \frac{\partial}{\partial x_{i}}\left(a_{i j}(x) \frac{\partial u}{\partial x_{j}}\right)=0 \quad \text { in } Q, \\
\left.u\right|_{\Gamma_{1}}=0,\left.\quad u\right|_{\Gamma_{0}}=c(t), \\
\int_{\Gamma_{0}} \frac{\partial u}{\partial n_{A}} d s=0, \quad u(x, T)=u(T) \quad \text { in } \Omega,
\end{gathered}
$$

where $u(T) \in L^{2}(\Omega)$, and similarly to system $(1.2), c(\cdot) \in L^{2}(0, T)$ is an unknown function. More precisely, we need to show the following.

Theorem 2.2. Under the assumption (2.1), there is a constant $C>0$ such that solutions $u \in Y$ of system (2.2) satisfy

$$
\|u(0)\|_{L^{2}(\Omega)} \leq C\|u(x, t)\|_{L^{2}(\omega \times(0, T))} .
$$

Remark 2.3. It would be quite interesting to drop the technical condition (2.1). But this is by now an unsolved problem.

\section{Proof of the main theorem}

It suffices to prove Theorem 2.2. To this end, for any given parameters $\lambda$ and $\mu$, we set

$$
\begin{aligned}
& \alpha(x, t)=(t(T-t))^{-1}\left(e^{\mu \psi(x)}-e^{2 \mu\|\psi\|_{C(\bar{\Omega})}}\right), \\
& \varphi(x, t)=(t(T-t))^{-1} e^{\mu \psi(x)}, \quad \theta(x, t)=e^{\lambda \alpha(x, t)} .
\end{aligned}
$$

Clearly, Theorem 2.2 is an easy consequence of the following global Carleman-type estimate for solutions of (2.2).

Theorem 3.1. Let (2.1) hold. Then there exist a constant $\mu_{1}$ and a function $\lambda_{1}: \mathbb{R}^{+} \rightarrow(1, \infty)$ such that for any $\mu>\mu_{1}$ and $\lambda>\lambda_{1}(\mu)$, solutions $u \in Y$ of system (2.2) satisfy

$$
\begin{aligned}
& \lambda^{3} \int_{Q} \theta^{2} \varphi^{3} u^{2} d x d t+\lambda \int_{Q} \theta^{2} \varphi|\nabla u|^{2} d x d t+\lambda^{-1} \int_{Q} \theta^{2} \varphi^{-1}\left[u_{t}^{2}+(\Delta u)^{2}\right] d x d t \\
& \quad \leq C \lambda^{3} \int_{Q^{\omega}} \theta^{2} \varphi^{3} u^{2} d x d t .
\end{aligned}
$$

The rest of this section is devoted to prove Theorem 3.1. For this, we need the following pointwise estimate for parabolic operator $L u$, which is a special case of [4, Lemma 3.1]. 
4 Null controllability of a nonlocal parabolic equation

Lemma 3.2. Let $u, \alpha \in C^{2}\left(\mathbb{R}^{n+1}\right)$ and $\lambda>0$ be given. Put $\theta=e^{\lambda \alpha}$ and $v=\theta u$. Then it holds that

$$
\begin{aligned}
& \theta^{2}|L u|^{2} \geq\left[-\lambda \alpha_{t} v^{2}-\sum_{i, j}\left(a_{i j} v_{x_{i}} v_{x_{j}}+\lambda\left(a_{i j}\right)_{x_{j}} \alpha_{x_{i}} v^{2}+\lambda a_{i j} \alpha_{x_{i} x_{j}} v^{2}-\lambda^{2} a_{i j} \alpha_{x_{i}} \alpha_{x_{j}} v^{2}\right)\right]_{t} \\
& +\sum_{j}\left[2 \sum_{i}\left(a_{i j} v_{x_{i}} v_{t}+\lambda^{2} a_{i j} \alpha_{x_{i}} \alpha_{t} v^{2}\right)\right. \\
& +2 \lambda \sum_{i}\left(a_{i j} a_{\ell m} \alpha_{x_{i}} v_{x_{\ell}} v_{x_{m}}+a_{i j}\left(a_{\ell m} \alpha_{x_{\ell} x_{m}}\right)_{x_{i}} v^{2}-2 a_{i j} a_{\ell m} \alpha_{x_{\ell}} v_{x_{i}} v_{x_{m}}\right. \\
& -2 a_{i j} a_{\ell m} \alpha_{x_{\ell} x_{m}} v v_{x_{i}}+\lambda a_{i j} a_{\ell m} \alpha_{x_{i}} \alpha_{x_{\ell} x_{m}} v^{2} \\
& \left.\left.+\lambda a_{i j}\left(a_{\ell m}\right)_{x_{m}} \alpha_{x_{\ell}} \alpha_{x_{m}} v^{2}-\lambda^{2} a_{i j} a_{\ell m} \alpha_{x_{\ell}} \alpha_{x_{m}} v^{2}\right)\right]_{x_{j}} \\
& +\left\{\lambda \alpha_{t t}-\lambda \sum_{i, j}\left[\left(\left(a_{i j}\right)_{x_{j}} \alpha_{x_{i}}\right)_{t}+\left(a_{i j} \alpha_{x_{i} x_{j}}\right)_{t}-\lambda\left(\alpha_{i j} \alpha_{x_{i}} \alpha_{x_{j}}\right)_{t}\right.\right. \\
& \left.-2 \lambda\left(a_{i j} \alpha_{x_{i}} \alpha_{t}\right)_{x_{j}}+4 \lambda a_{i j} \alpha_{x_{i} x_{j}} \alpha_{t}\right] \\
& +2 \lambda \sum_{i, j, \ell, m}\left[2 \lambda\left(a_{i j}\right)_{x_{j}} a_{\ell m} \alpha_{x_{i}} \alpha_{x_{\ell} x_{m}}-\left(a_{i j}\left(a_{\ell m} \alpha_{x_{\ell} x_{m}}\right)_{x_{j}}\right)_{x_{i}}-\lambda\left(\left(a_{\ell m}\right)_{x_{m}} a_{i j} \alpha_{x_{i}} \alpha_{x_{\ell}}\right)_{x_{j}}\right. \\
& -\lambda\left(a_{i j} a_{\ell m} \alpha_{x_{i} x_{j}} \alpha_{x_{\ell}}\right)_{x_{m}}-2 \lambda a_{i j} a_{\ell m} \alpha_{x_{i} x_{j}} \alpha_{x_{\ell} x_{m}} \\
& \left.\left.+\lambda^{2}\left(a_{i j} a_{\ell m} \alpha_{x_{i}} \alpha_{x_{j}} \alpha_{x_{\ell}}\right)_{x_{m}}-2 \lambda^{2} a_{i j} a_{\ell m} \alpha_{x_{i}} \alpha_{x_{j}} \alpha_{x_{\ell} x_{m}}\right]\right\} v^{2} \\
& +2 \lambda \sum_{i, j, \ell, m}\left[2 a_{i j}\left(a_{\ell m} \alpha_{x_{\ell}}\right)_{x_{j}} v_{x_{i}} v_{x_{m}}-\left(a_{i j} a_{\ell m}\right)_{x_{m}} \alpha_{x_{\ell}} v_{x_{i}} v_{x_{j}}+a_{i j} a_{\ell m} \alpha_{x_{\ell} x_{m}} v_{x_{i}} v_{x_{j}}\right] \text {. }
\end{aligned}
$$

Proof of Theorem 3.1. The main idea of our proof is to use the pointwise estimate in Lemma 3.2. The proof is divided into several steps.

Step 1. Recall that $\theta=e^{\lambda \alpha}, v=\theta u$. We claim that

$$
\begin{gathered}
\int_{Q} \theta^{2}(L u)^{2} d x d t-\int_{Q} \operatorname{div} V d x d t+C \lambda^{3} \mu^{3} \int_{Q} \varphi^{3} v^{2} d x d t+C \lambda \mu^{2} \int_{Q} \varphi|\nabla v|^{2} d x d t \\
\geq 2 \Lambda^{2} \lambda^{3} \mu^{4} \int_{Q} \varphi^{3}|\nabla \psi|^{4} v^{2} d x d t+2 \Lambda^{2} \lambda \mu^{2} \int_{Q} \varphi|\nabla v|^{2}|\nabla \psi|^{2} d x d t,
\end{gathered}
$$

where $V=\left(V_{1}, V_{2}, \ldots, V_{n}\right)$, and

$$
\begin{gathered}
V_{j}=-2 \sum_{i}\left(a_{i j} v_{x_{i}} v_{t}+\lambda^{2} a_{i j} \alpha_{x_{i}} \alpha_{t} v^{2}\right) \\
+2 \lambda \sum_{i, \ell, m}\left(a_{i j} a_{\ell m} \alpha_{x_{i}} v_{x_{\ell}} v_{x_{m}}-2 a_{i j} a_{\ell m} \alpha_{x_{\ell}} v_{x_{i}} v_{x_{m}}+\lambda a_{i j}\left(a_{\ell m}\right)_{x_{m}} \alpha_{x_{i}} \alpha_{x_{m}} v^{2}\right. \\
-\lambda^{2} a_{i j} a_{\ell m} \alpha_{x_{i}} \alpha_{x_{\ell}} \alpha_{x_{m}} v^{2}+\lambda a_{i j} a_{\ell m} \alpha_{x_{i}} \alpha_{x_{\ell} x_{m}} v^{2} \\
\left.-2 a_{i j} a_{\ell m} \alpha_{x_{\ell} x_{m}} v v_{x_{i}}+a_{i j}\left(a_{\ell m} \alpha_{x_{\ell} x_{m}}\right)_{x_{i}} v^{2}\right)
\end{gathered}
$$


By the definition of $\alpha$ and $\varphi$ in (3.1), it is easy to see that

$$
\int_{Q}\left[-\lambda \alpha_{t} v^{2}+\sum_{i, j}\left(a_{i j} v_{x_{i}} v_{x_{j}}+\lambda\left(a_{i j}\right)_{x_{j}} \alpha_{x_{i}} v^{2}-\lambda^{2} a_{i j} \alpha_{x_{i}} \alpha_{x_{j}}^{2}+\lambda a_{i j} \alpha_{x_{i} x_{j}} v^{2}\right)\right]_{t} d t=0
$$

Let us estimate the last three "energy" terms of first order in the right-hand side of (3.3). First,

$$
\begin{aligned}
& \left|\int_{Q} 4 \lambda \sum_{i, j, \ell, m} a_{i j}\left(a_{\ell m} \alpha_{x_{\ell}}\right)_{x_{j}} v_{x_{i}} v_{x_{m}} d x d t\right| \\
& =\left|\int_{Q} 4 \lambda \sum_{i, j, \ell, m}\left(a_{i j}\left(a_{\ell m}\right)_{x_{j}} \mu \psi_{x_{\ell}} \varphi v_{x_{i}} v_{x_{m}}+a_{i j} a_{\ell m}\left(\mu \psi_{x_{\ell} x_{j}} \varphi+\mu^{2} \psi_{x_{\ell}} \psi_{x_{j}} \varphi\right) v_{x_{i}} v_{x_{m}}\right) d x d t\right| \\
& \quad \leq C \lambda \mu \int_{Q} \varphi|\nabla v|^{2} d x d t+C \lambda \mu^{2} \int_{Q} \varphi|\nabla v|^{2} d x d t
\end{aligned}
$$

where $C$ is a positive constant for $\lambda$ large enough.

Next, it is easy to see that

$$
\begin{gathered}
\left|-\int_{Q} 2 \lambda \sum_{i, j, \ell, m}\left(a_{i j} a_{\ell m}\right)_{x_{m}} \alpha_{x_{\ell}} v_{x_{i}} v_{x_{j}} d x d t\right| \leq C \lambda \mu \int_{Q} \varphi|\nabla v|^{2} d x d t \\
\int_{Q} 2 \lambda \sum_{i, j, \ell, m} a_{i j} a_{\ell m} \alpha_{x_{\ell} x_{m}} v_{x_{i}} v_{x_{j}} d x d t \\
=\int_{Q} 2 \lambda \sum_{i, j, \ell, m}\left(\mu a_{i j} a_{\ell m} \psi_{x_{\ell} x_{m}} \varphi v_{x_{i}} v_{x_{j}}+\mu^{2} a_{i j} a_{\ell m} \psi_{x_{\ell}} \psi_{x_{m}} \varphi v_{x_{i}} v_{x_{j}}\right) d x d t \\
\geq-C \lambda \mu \int_{Q} \varphi|\nabla v|^{2} d x d t+2 \lambda \mu^{2} \Lambda^{2} \int_{Q} \varphi|\nabla \psi|^{2}|\nabla v|^{2} d x d t .
\end{gathered}
$$

It remains to deal with the "energy" term of zero order, that is, $\int_{Q}\{\cdots\} v^{2} d x d t$, in the right-hand side of (3.3). Similarly to [4], we have

$$
\begin{gathered}
-2 \int_{2} \lambda^{3} \sum_{i, j, \ell, m}\left(2 a_{i j} a_{\ell m} \alpha_{x_{i}} \alpha_{x_{j}} \alpha_{x_{\ell} x_{m}} v^{2}-\left(a_{i j} a_{\ell m} \alpha_{x_{i}} \alpha_{x_{j}} \alpha_{x_{\ell}}\right)_{x_{m}} v^{2}\right) d x d t \\
=-\int_{2} 2 \lambda^{3} \mu^{3} \sum_{i, j, \ell, m}\left(2 a_{i j} a_{\ell m} \psi_{x_{i}} \psi_{x_{j}} \psi_{x_{\ell} x_{m}} \varphi^{3} v^{2}+2 \mu a_{i j} a_{\ell m} \psi_{x_{i}} \psi_{x_{j}} \psi_{x_{\ell}} \psi_{x_{m}} \varphi^{3} v^{2}\right. \\
\left.-\left(a_{i j} a_{\ell m} \psi_{x_{i}} \psi_{x_{j}} \psi_{x_{\ell}}\right)_{x_{m}} \varphi^{3} v^{2}-3 \mu a_{i j} a_{\ell m} \psi_{x_{i}} \psi_{x_{j}} \psi_{x_{\ell}} \psi_{x_{m}} \varphi^{3} v^{2}\right) d x d t \\
\geq-C \lambda^{3} \mu^{3} \int_{2} \varphi^{3} v^{2} d x d t+2 \lambda^{3} \mu^{4} \Lambda^{2} \int_{2} \varphi^{3}|\nabla \psi|^{4} v^{2} d x d t .
\end{gathered}
$$


6 Null controllability of a nonlocal parabolic equation

Therefore, for large $\lambda$, the following estimate holds:

$$
\begin{gathered}
\int_{Q}\left\{\lambda \alpha_{t t}-\lambda \sum_{i, j}\left[\left(\left(a_{i j}\right)_{x_{j}} \alpha_{x_{i}}\right)_{t}-\lambda\left(a_{i j} \alpha_{x_{i}} \alpha_{x_{j}}\right)_{t}+\left(a_{i j} \alpha_{x_{i} x_{j}}\right)_{t}-2 \lambda\left(a_{i j} \alpha_{x_{i}} \alpha_{t}\right)_{x_{j}}+4 \lambda a_{i j} \alpha_{x_{i} x_{j}} \alpha_{t}\right]\right. \\
+2 \lambda \sum_{i, j, \ell, m}\left[\lambda^{2}\left(a_{i j} a_{\ell m} \alpha_{x_{i}} \alpha_{x_{j}} \alpha_{x_{\ell}}\right)_{x_{m}}-\lambda\left(a_{i j} a_{\ell m} \alpha_{x_{i} x_{j}} \alpha_{x_{\ell}}\right)_{x_{m}}-\lambda\left(\left(a_{\ell m}\right)_{x_{m}} a_{i j} \alpha_{x_{i}} \alpha_{x_{j}}\right)_{x_{j}}\right. \\
+2 \lambda\left(a_{i j}\right)_{x_{j}} a_{\ell m} \alpha_{x_{i}} \alpha_{x_{\ell} x_{m}}-2 \lambda^{2} a_{i j} a_{\ell m} \alpha_{x_{i}} \alpha_{x_{j}} \alpha_{x_{\ell} x_{m}} \\
\left.\left.-2 \lambda a_{i j} a_{\ell m} \alpha_{x_{i} x_{j}} \alpha_{x_{\ell} x_{m}}-\left(a_{i j}\left(a_{\ell m} \alpha_{x_{\ell} x_{m}}\right)_{x_{j}}\right)_{x_{i}}\right]\right\} v^{2} d x d t \\
\geq-C \lambda^{3} \mu^{3} \int_{2} \varphi^{3} v^{2} d x d t+2 \lambda^{3} \mu^{4} \Lambda^{2} \int_{Q} \varphi^{3}|\nabla \psi|^{4} v^{2} d x d t .
\end{gathered}
$$

Step 2. From (3.4), one finds

$$
\begin{gathered}
C\left(\int_{Q} \theta^{2}(L u)^{2} d x d t-\int_{Q} \operatorname{div} V d x d t+\lambda^{3} \mu^{3} \int_{Q} \varphi^{3} v^{2} d x d t+\lambda \mu^{2} \int_{Q} \varphi|\nabla v|^{2} d x d t\right) \\
\geq \lambda^{3} \mu^{4} \int_{Q} \varphi^{3}|\nabla \psi|^{4} v^{2} d x d t+\lambda \mu^{2} \int_{Q} \varphi|\nabla \psi|^{2}|\nabla v|^{2} d x d t .
\end{gathered}
$$

Set

$$
Q^{\omega_{0}}=\omega_{0} \times(0, T)
$$

Noting that $|\nabla \psi(x)|>0$ for all $x \in \Omega_{0}=\bar{\Omega} \backslash \omega_{0}$, by (3.11), it is easy to see that

$$
\begin{gathered}
C\left(\int_{Q} \theta^{2}(L u)^{2} d x d t-\int_{Q} \operatorname{div} V d x d t+\lambda^{3} \mu^{3} \int_{Q^{\omega_{0}}} \varphi^{3} v^{2} d x d t+\lambda \mu^{2} \int_{Q^{\omega_{0}}} \varphi|\nabla v|^{2} d x d t\right) \\
\quad \geq \lambda^{3} \mu^{4} \int_{Q} \varphi^{3} v^{2} d x d t+\lambda \mu^{2} \int_{Q} \varphi|\nabla v|^{2} d x d t .
\end{gathered}
$$

Returning $v$ to $e^{\lambda \alpha} u$ in (3.13), we arrive at

$$
\begin{aligned}
& C\left(\int_{Q} \theta^{2}(L u)^{2} d x d t-\int_{Q} \operatorname{div} V d x d t+\lambda^{3} \int_{Q^{\omega_{0}}} \theta^{2} \varphi^{3} u^{2} d x d t+\lambda \int_{Q^{\omega_{0}}} \theta^{2} \varphi|\nabla u|^{2} d x d t\right) \\
& \quad \geq \lambda^{3} \int_{Q} \theta^{2} \varphi^{3} u^{2} d x d t+\lambda \int_{Q} \theta^{2} \varphi|\nabla u|^{2} d x d t
\end{aligned}
$$

Step 3. The purpose of this step is to get rid of the fourth term in the left-hand side of (3.14). To this end, we multiply $L u$ by $\tilde{\chi} \theta^{2} \varphi u$ and then integrate it over $Q$, where $\tilde{\chi} \in$ $C_{0}^{\infty}(\omega), \tilde{\chi}=1$ in $\omega_{0}$, and $\tilde{\chi}=0$ in $\Omega \backslash \omega$. Then, we obtain

$$
\int_{Q} \frac{\partial u}{\partial t} \tilde{\chi} \theta^{2} \varphi u d x d t+\int_{Q} \sum_{i, j} \frac{\partial}{\partial x_{i}}\left(a_{i j}(x) \frac{\partial u}{\partial x_{j}}\right) \tilde{\chi} \theta^{2} \varphi u d x d t=\int_{Q}(L u) \tilde{\chi} \theta^{2} \varphi u d x d t .
$$


Using integration by parts, it is easy to deduce from (3.15) that

$$
\lambda \int_{Q^{\omega_{0}}} \theta^{2} \varphi|\nabla u|^{2} d x d t \leq C\left(\int_{Q} \theta^{2}(L u)^{2} d x d t+\lambda^{3} \int_{Q^{\omega}} \theta^{2} \varphi^{3} u^{2} d x d t\right) .
$$

Combining (3.13) and (3.16), we end up with

$$
\begin{gathered}
C\left(\int_{Q} \theta^{2}(L u)^{2} d x d t+\lambda^{3} \int_{Q^{\omega}} \theta^{2} \varphi^{3} u^{2} d x d t-\int_{Q} \operatorname{div} V d x d t\right) \\
\geq \lambda^{3} \int_{Q} \theta^{2} \varphi^{3} u^{2} d x d t+\lambda \int_{Q} \theta^{2} \varphi|\nabla u|^{2} d x d t
\end{gathered}
$$

Step 4. This step is to estimate the "divergence" term div $V$. Denote the terms on the right-hand side of (3.5) by $I_{i}, i=1,2, \ldots, 9$. First,

$$
\begin{aligned}
I_{1}+I_{2} & =\int_{Q} \sum_{j}\left(2 \sum_{i}\left(a_{i j} v_{x_{i}} v_{t}+\lambda^{2} a_{i j} \alpha_{x_{i}} \alpha_{t} v^{2}\right)\right)_{x_{j}} \\
& =\lambda^{2} \mu \int_{0}^{T} \theta^{2} \varphi_{t} c^{2}(t) d t \int_{\Gamma_{0}} \sum_{i, j} a_{i j} \psi_{x_{i}} n_{j} d s-2 \lambda^{2} \mu \int_{0}^{T} \theta^{2} \varphi \alpha_{t} c^{2}(t) d t \int_{\Gamma_{0}} \sum_{i, j} a_{i j} \psi_{x_{i}} n_{j} d s .
\end{aligned}
$$

Next,

$$
\begin{aligned}
I_{3}+I_{5}+I_{9}= & \int_{Q} \sum_{j}\left(2 \lambda \sum_{i, \ell, m}\left(a_{i j} a_{\ell m} \alpha_{x_{i}} v_{x_{\ell}} v_{x_{m}}-2 a_{i j} a_{\ell m} \alpha_{x_{\ell}} v_{x_{i}} v_{x_{m}}-\lambda^{2} a_{i j} a_{\ell m} \alpha_{x_{i}} \alpha_{x_{m}} v^{2}\right)\right)_{x_{j}} d x d t \\
= & -4 \lambda^{3} \mu^{3} \int_{0}^{T} \varphi^{3} c^{2}(t) \theta^{2} d t \int_{\Gamma_{0}} \sum_{i, j, \ell, m} a_{i j} a_{\ell m} \psi_{x_{i}} \psi_{x_{\ell}} \psi_{x_{m}} n_{j} d s \\
& -4 \lambda^{2} \mu^{2} \int_{0}^{T} \varphi^{2} \theta^{2} c(t) d t \int_{\Gamma_{0}} \sum_{i, j, \ell, m} a_{i j} a_{\ell m} \psi_{x_{\ell}} \psi_{x_{i}} u_{x_{m}} n_{j} d s \\
& -2 \int_{0}^{T} \lambda \mu \varphi \theta^{2} d t \int_{\Gamma_{i, j, \ell, m}} a_{i j} a_{\ell m} \psi_{x_{\ell}} u_{x_{i}} u_{x_{m}} n_{j} d s .
\end{aligned}
$$

Further,

$$
\begin{aligned}
I_{4}= & \int_{Q} \sum_{j}\left(2 \lambda \sum_{i} a_{i j}\left(a_{\ell m} \alpha_{x_{\ell} x_{m}}\right)_{x_{i}} v^{2}\right)_{x_{j}} d x d t \\
= & 2 \lambda \mu^{2} \int_{0}^{T} \theta^{2} \varphi c^{2}(t) d t \int_{\Gamma_{0}} \sum_{i, j, \ell, m} a_{i j}\left(a_{\ell m}\right)_{x_{i}} \psi_{x_{\ell}} \psi_{x_{m}} n_{j} d s \\
& +2 \lambda \mu \int_{0}^{T} \theta^{2} \varphi c^{2}(t) d t \int_{\Gamma_{0}} \sum_{i, j, \ell, m} a_{i j}\left(a_{\ell m}\right)_{x_{i}} \psi_{x_{\ell} x_{m}} n_{j} d s \\
& +2 \lambda \mu^{3} \int_{0}^{T} \theta^{2} \varphi c^{2}(t) d t \int_{\Gamma_{0}} \sum_{i, j, \ell, m} a_{i j} a_{\ell m} \psi_{x_{i}} \psi_{x_{\ell}} \psi_{x_{m}} n_{j} d s
\end{aligned}
$$


8 Null controllability of a nonlocal parabolic equation

$$
\begin{aligned}
& +2 \lambda \mu^{2} \int_{0}^{T} \theta^{2} \varphi c^{2}(t) d t \int_{\Gamma_{0}} \sum_{i, j, \ell, m} a_{i j} a_{\ell m} \psi_{x_{i} x_{\ell}} \psi_{x_{m}} n_{j} d s \\
& +2 \lambda \mu^{2} \int_{0}^{T} \theta^{2} \varphi c^{2}(t) d t \int_{\Gamma_{0}} \sum_{i, j, \ell, m} a_{i j} a_{\ell m} \psi_{x_{\ell}} \psi_{x_{i} x_{m}} n_{j} d s \\
& +2 \lambda \mu \int_{0}^{T} \theta^{2} \varphi c^{2}(t) d t \int_{\Gamma_{0}} \sum_{i, j, \ell, m} a_{i j} a_{\ell m} \psi_{x_{i} x_{\ell} x_{\ell}} n_{j} d s \\
& \geq C \lambda \mu^{3} \int_{0}^{T} \theta^{2} \varphi c^{2}(t) d t \int_{\Gamma_{0}} \sum_{i, j, \ell, m} a_{i j} a_{\ell m} \psi_{x_{i}} \psi_{x_{\ell}} \psi_{x_{m}} n_{j} d s .
\end{aligned}
$$

Further,

$$
\begin{aligned}
I_{6}= & -\int_{Q} \sum_{j}\left(4 \lambda \sum_{i, \ell, m} a_{i j} a_{\ell m} \alpha_{x_{\ell} x_{m}} v v_{x_{i}}\right)_{x_{j}} d x d t \\
= & -4 \lambda^{2} \mu^{3} \int_{0}^{T} \theta^{2} \varphi^{2} c^{2}(t) d t \int_{\Gamma_{0}} \sum_{i, j, \ell, m} a_{i j} a_{\ell m} \psi_{x_{i}} \psi_{x_{\ell}} \psi_{x_{m}} n_{j} d s \\
& -4 \lambda \mu^{2} \int_{0}^{T} \theta^{2} \varphi^{2} c^{2}(t) d t \int_{\Gamma_{0}} \sum_{i, j, \ell, m} a_{i j} a_{\ell m} \psi_{x_{i}} \psi_{x_{\ell} x_{m}} n_{j} d s \\
& -4 \lambda \mu^{2} \int_{0}^{T} \theta^{2} \varphi c(t) d t \int_{\Gamma_{0}} \sum_{i, j, \ell, m} a_{i j} a_{\ell m} \psi_{x_{\ell}} \psi_{x_{m}} u_{x_{i}} n_{j} d s \\
& -4 \lambda \mu \int_{0}^{T} \theta^{2} \varphi c(t) d t \int_{\Gamma_{0}} \sum_{i, j, \ell, m} a_{i j} a_{\ell m} \psi_{x_{\ell} x_{m}} u_{x_{i}} n_{j} d s .
\end{aligned}
$$

Finally,

$$
\begin{aligned}
I_{7}+I_{8}= & \int_{Q} \sum_{j}\left(2 \lambda^{2} \sum_{i, \ell, m}\left(a_{i j} a_{\ell m} \alpha_{x_{i}} \alpha_{x_{\ell} x_{m}} v^{2}+a_{i j}\left(a_{\ell m}\right)_{x_{m}} \alpha_{x_{i}} \alpha_{x_{m}} v^{2}\right)\right)_{x_{j}} d x d t \\
= & 2 \lambda^{2} \mu^{3} \int_{0}^{T} \theta^{2} \varphi^{2} c^{2}(t) d t \int_{\Gamma_{0}} \sum_{i, j, \ell, m} a_{i j} a_{\ell m} \psi_{x_{i}} \psi_{x_{\ell}} \psi_{x_{m}} n_{j} d s \\
& +2 \lambda^{2} \mu^{2} \int_{0}^{T} \theta^{2} \varphi^{2} c^{2}(t) d t \int_{\Gamma_{0}} \sum_{i, j, \ell, m} a_{i j} a_{\ell m} \psi_{x_{i}} \psi_{x_{\ell} x_{m}} n_{j} d s \\
& +2 \lambda^{2} \mu^{2} \int_{0}^{T} \theta^{2} \varphi^{2} c^{2}(t) d t \int_{\Gamma_{0}} \sum_{i, j, \ell, m} a_{i j}\left(a_{\ell m}\right)_{x_{m}} \psi_{x_{i}} \psi_{x_{m}} n_{j} d s .
\end{aligned}
$$

Now, combining (3.18)-(3.22) and using the technical condition (2.1), we conclude that, for large $\lambda$ and $\mu$, it holds that

$$
\int_{Q} \operatorname{div} V d x d t \geq C \lambda^{3} \mu^{3} \int_{0}^{T} \theta^{2} \varphi^{3} c^{2}(t) d t-4 \lambda \mu \Lambda \int_{0}^{T} \theta^{2} \varphi d t \int_{\Gamma}|\nabla u|^{2} \sum_{i, j} a_{i j} \psi_{x_{i}} n_{j} d s .
$$


Step 5. It remains to estimate $\int_{Q}(\lambda \varphi)^{-1} \theta^{2}\left(u_{t}^{2}+\sum_{i, j}\left(a_{i j} u_{x_{i}}\right)_{x_{j}}\right)^{2} d x d t$. For this, we observe that

$$
\begin{aligned}
\left(v_{t}+\sum_{i, j}\left(a_{i j} v_{x_{i}}\right)_{x_{j}}\right)^{2} \leq C & {\left[\lambda^{2} \alpha_{t}^{2} v^{2}+\sum_{i, j}\left(\lambda^{2}\left(a_{i j}\right)_{x_{j}}^{2} \alpha_{x_{i}}^{2} v^{2}+\lambda^{2} a_{i j}^{2} \alpha_{x_{i} x_{j}}^{2} v^{2}\right.\right.} \\
& \left.\left.+\lambda^{4} a_{i j}^{2} \alpha_{x_{i}}^{2} \alpha_{x_{j}}^{2} v^{2}+\lambda^{2} a_{i j}^{2} \alpha_{i j}^{2} \alpha_{x_{i}}^{2} v_{x_{j}}^{2}+\left(a_{i j}\right)_{x_{j}}^{2} v_{x_{i}}^{2}\right)\right] .
\end{aligned}
$$

This implies

$$
\begin{gathered}
(\lambda \varphi)^{-1}\left(v_{t}+\sum_{i, j}\left(a_{i j} v_{x_{i}}\right)_{x_{j}}\right)^{2} \\
\leq C(\lambda \varphi)^{-1}\left[\lambda^{2} \alpha_{t}^{2} v^{2}+\sum_{i, j}\left(\lambda^{2}\left(a_{i j}\right)_{x_{j}}^{2} \alpha_{x_{i}}^{2} v^{2}+\lambda^{2} a_{i j}^{2} \alpha_{x_{i} x_{j}}^{2} v^{2}\right.\right. \\
\left.\left.+\lambda^{4} a_{i j}^{2} \alpha_{x_{i}}^{2} \alpha_{x_{j}}^{2} v^{2}+\lambda^{2} a_{i j}^{2} \alpha_{i j}^{2} \alpha_{x_{i}}^{2} v_{x_{j}}^{2}+\left(a_{i j}\right)_{x_{j}}^{2} v_{x_{i}}^{2}\right)\right] \\
\leq C\left(\lambda^{3} \mu^{4} \varphi^{3} v^{2}+\lambda \mu \varphi|\nabla v|^{2}\right) .
\end{gathered}
$$

Noting that

$$
\begin{aligned}
& 2 \int_{Q} \sum_{i, j}(\lambda \varphi)^{-1} v_{t}\left(a_{i j} v_{x_{i}}\right)_{x_{j}} d x d t \\
&=2 \sum_{i, j}\left(\int_{Q}\left(a_{i j}(\lambda \varphi)^{-1} v_{x_{i}} v_{t}\right)_{x_{j}} d x d t-2 \int_{Q} a_{i j}(\lambda \varphi)_{x_{j}}^{-1} v_{x_{i}} v_{t} d x d t+\int_{Q} a_{i j}(\lambda \varphi)_{t}^{-1} v_{x_{i}} v_{x_{j}} d x d t\right. \\
&\left.\quad+\int_{Q}\left(a_{i j}\right)_{t}(\lambda \varphi)^{-1} v_{x_{i}} v_{x_{j}} d x d t+\int_{Q} a_{i j}\left((\lambda \varphi)^{-1} v_{t}\right)_{x_{i}} v_{x_{j}} d x d t\right),
\end{aligned}
$$

we get

$$
\begin{aligned}
2 \int_{Q}(\lambda \varphi)^{-1} \sum_{i, j}\left(a_{i j} v_{x_{i}}\right)_{x_{j}} v_{t} d x d t \leq & \frac{1}{2} \int_{Q}(\lambda \varphi)^{-1} v_{t}^{2} d x d t+C \lambda \int_{Q} \varphi|\nabla v|^{2} d x d t \\
& +\lambda \mu \int_{0}^{T} \theta \alpha_{t} c^{2}(t) d t \int_{\Gamma_{0}} \sum_{i, j} a_{i j} \psi_{x_{i}} n_{j} d s .
\end{aligned}
$$

By (3.24)-(3.27), we obtain that

$$
\begin{aligned}
& \int_{Q}(\lambda \varphi)^{-1}\left[v_{t}^{2}+\left(v_{x_{i} x_{j}}\right)^{2}\right] d x d t \\
& \leq C\left(\int_{Q}(L u)^{2} \theta^{2} d x d t+\lambda^{3} \int_{Q^{\omega_{0}}} \mu^{3} \varphi^{3} v^{2} d x d t+\lambda \int_{Q^{\omega_{0}}} \mu \varphi|\nabla v|^{2} d x d t\right) \\
&+\lambda \mu \int_{0}^{T} \theta \alpha_{t} c^{2}(t) d t \int_{\Gamma_{0}} \sum_{i, j} a_{i j} \psi_{x_{i}} n_{j} d s .
\end{aligned}
$$


This gives

$$
\begin{aligned}
& \int_{Q}(\lambda \varphi)^{-1}\left[v_{t}^{2}+(\Delta v)^{2}\right] d x d t \\
& \leq C\left(\int_{Q}(L u)^{2} \theta^{2} d x d t+\lambda^{3} \int_{Q^{\omega_{0}}} \mu^{3} \varphi^{3} v^{2} d x d t+\lambda \int_{Q^{\omega_{0}}} \mu \varphi|\nabla v|^{2} d x d t\right) \\
&+\lambda \mu \int_{0}^{T} \theta \alpha_{t} c^{2}(t) d t \int_{\Gamma_{0}} \sum_{i, j} a_{i j} \psi_{x_{i}} n_{j} d s .
\end{aligned}
$$

It is easy to see that the last term of the above inequality can be absorbed by (3.23) for large $\lambda$ and $\mu$. Finally, replacing $v$ by $e^{\lambda \alpha} u$ and combining (2.2), (3.16), (3.17), (3.23), and (3.29), we get the desired estimate (3.2). This completes the proof of Theorem 3.1.

\section{Acknowledgments}

This work is partially supported by FANEDD of China (Project No 200119), NCET of China under Grant NCET-04-0882, and the NSF of China under Grants 10371084 and 10525105. The author gratefully acknowledges Professor Xu Zhang for his guidance, encouragement, and suggestions.

\section{References}

[1] V. Barbu, Exact controllability of the superlinear heat equation, Applied Mathematics and Optimization. An International Journal with Applications to Stochastics 42 (2000), no. 1, 73-89.

[2] A. Doubova, E. Fernández-Cara, M. González-Burgos, and E. Zuazua, On the controllability of parabolic systems with a nonlinear term involving the state and the gradient, SIAM Journal on Control and Optimization 41 (2002), no. 3, 798-819.

[3] A. V. Fursikov and O. Yu. Imanuvilov, Controllability of Evolution Equations, Lecture Notes Series, vol. 34, Seoul National University, Research Institute of Mathematics, Global Analysis Research Center, Seoul, 1996.

[4] W. Li, Observability estimate for the ultra-parabolic equations, Master's thesis, School of Mathematics, Sichuan University, Chengdu, 2004.

[5] T. Li, S. Zheng, Y. Tan, and W. Shen, Boundary Value Problems with Equivalued Surface and Resistivity Well-Logging, Pitman Research Notes in Mathematics Series, vol. 382, Longman, Harlow, 1998.

[6] W. Shen, Initial-boundary problem for the second order parabolic equations with equivalued surface boundary condition, Journal of Fudan University Natural Science 4 (1978), 15-23 (Chinese).

[7] G. Wang and L. Wang, The Carleman inequality and its application to periodic optimal control governed by semilinear parabolic differential equations, Journal of Optimization Theory and Applications 118 (2003), no. 2, 429-461.

[8] X. Zhang, Solvability of non-linear parabolic boundary value problem with equivalued surface, Mathematical Methods in the Applied Sciences 22 (1999), no. 3, 259-265.

[9] __ Exact Controllability of Semi-linear Distributed Parameter Systems, Chinese Higher Education Press, Beijing, 2004.

Zhongqi Yin: Department of Engineering and Technology of Caotang School,

Sichuan Normal University, Chengdu 610072, China

E-mail address: zhongqiyin@sohu.com 


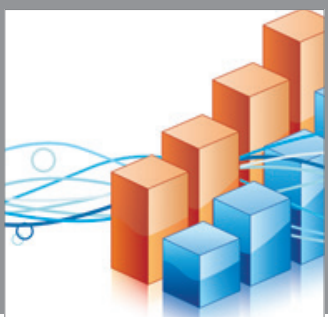

Advances in

Operations Research

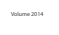

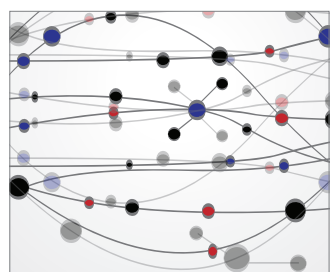

\section{The Scientific} World Journal
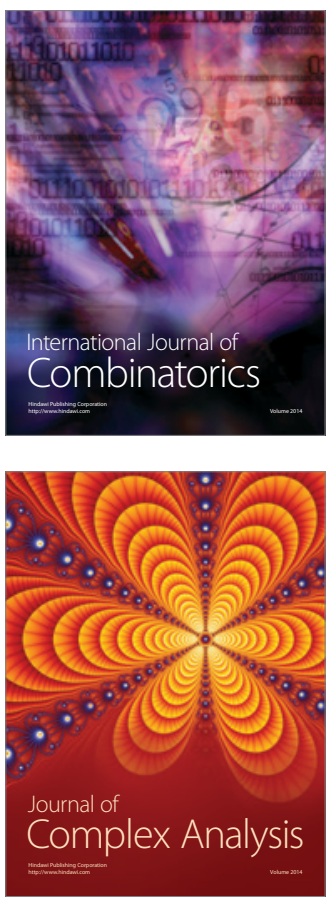

International Journal of

Mathematics and

Mathematical

Sciences
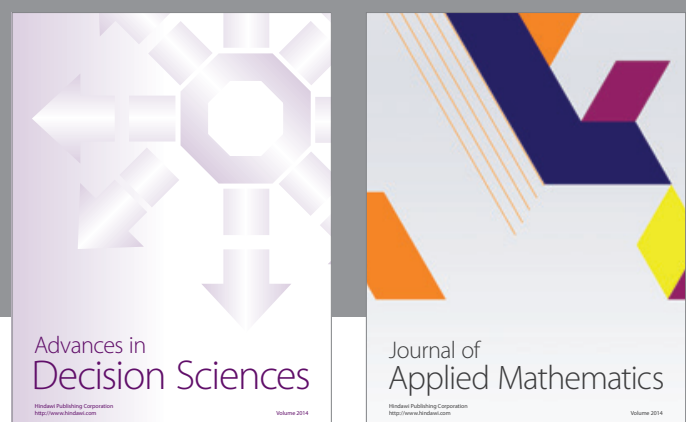

Journal of

Applied Mathematics
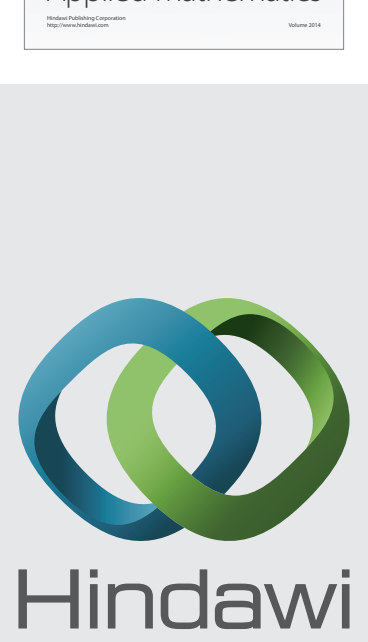

Submit your manuscripts at http://www.hindawi.com
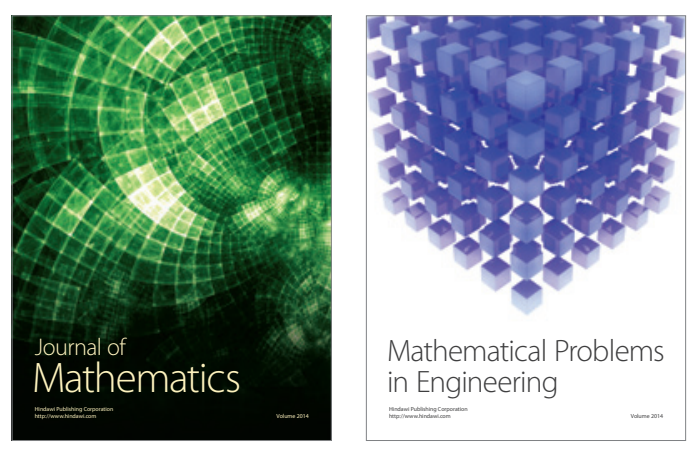

Mathematical Problems in Engineering
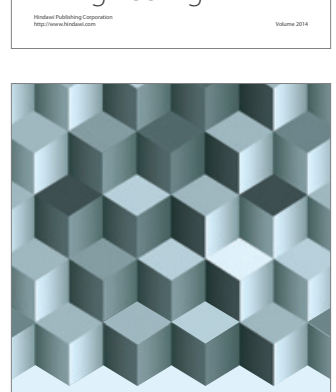

Journal of

Function Spaces
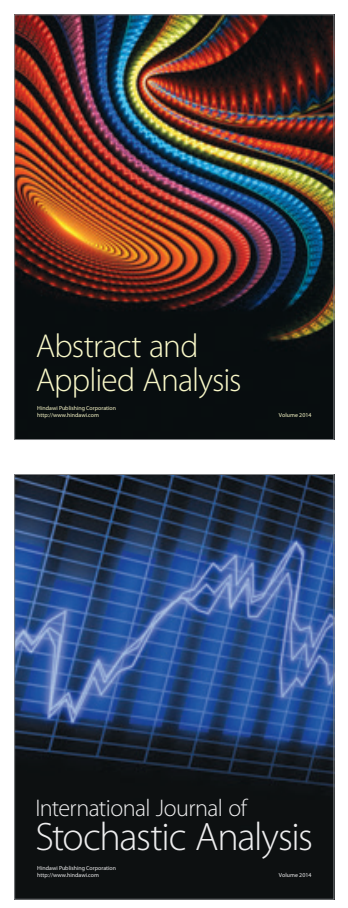

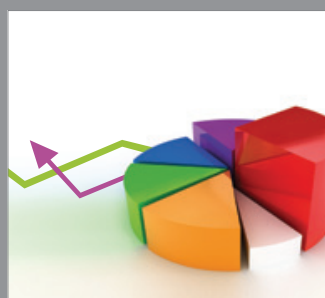

ournal of

Probability and Statistics

Promensencen
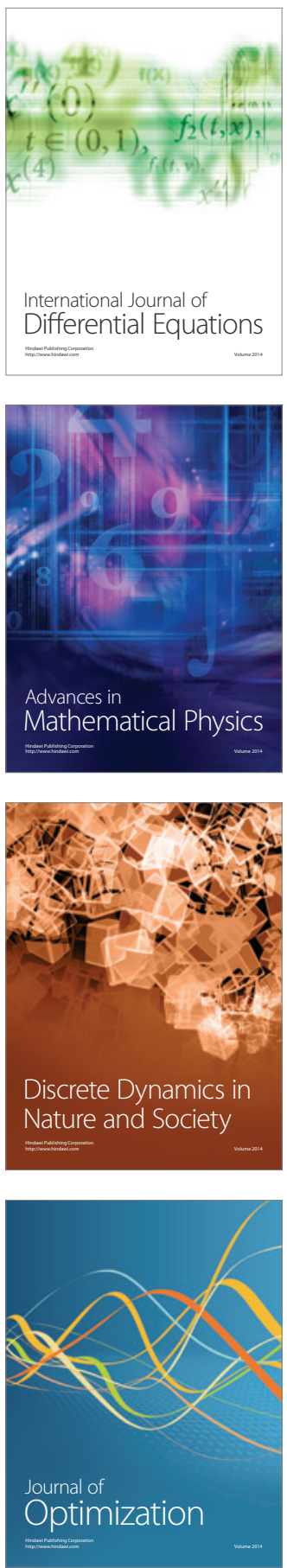